\title{
Modelling of a CFD Microscale Model and Its Application in Wind Energy Resource Assessment
}

\author{
Jie-shun Yue ${ }^{1, a}$, Song-ping $\mathrm{Wu}^{1,2}$ and Fei-shi $\mathrm{Xu}^{3}$ \\ ${ }^{1}$ School of Aeronautical Science and Engineering, Beihang University, 100083, Beijing, P. R. China \\ ${ }^{2}$ National computational fluid dynamics laboratory of China, 100083, Beijing, P. R. China \\ ${ }^{3}$ Sino-French Engineering School, Beihang University, 100083, Beijing, P. R. China
}

\begin{abstract}
The prediction of a wind farm near the wind turbines has a significant effect on the safety as well as economy of wind power generation. To assess the wind resource distribution within a complex terrain, a computational fluid dynamics (CFD) based wind farm forecast microscale model is developed. The model uses the Reynolds Averaged Navier-Stokes (RANS) model to characterize the turbulence. By using the results of Weather Research and Forecasting (WRF) mesoscale weather forecast model as the input of the CFD model, a coupled model of CFD-WRF is established. A special method is used for the treatment of the information interchange on the lateral boundary between two models. This established coupled model is applied in predicting the wind farm near a wind turbine in Hong Gang-zi, Jilin, China. The results from this simulation are compared to real measured data. On this basis, the accuracy and efficiency of turbulence characterization schemes are discussed. It indicates that this coupling system is easy to implement and can make these two separate models work in parallel. The CFD model coupled with WRF has the advantage of high accuracy and fast speed, which makes it valid for the wind power generation.
\end{abstract}

\section{Introduction}

Energy is one of the most valuable consumables of human society. With energy shortage becoming obvious, the demand for renewable energy is rapidly increasing. Among these available new energy resources is wind power which has widely been used during recent years. However, drastic fluctuations of wind power generation being caused by random wind variation will result in a serious strike on the power grid [1]. The reliable and accurate prediction of wind farm near the wind turbines can effectively improve the safety and economy for wind power generation. Currently, the most general method for wind farm prediction is using the mesoscale weather forecast models, e.g. WRF, MM5 [2]. The demand for a more precise prediction of atmospheric processes calls for the improvement of the resolution of model and grid. But it will cause extreme increase of computation and time expense if the fine mesh model is used with higher resolution in the whole prediction area and consequently making the model unable to meet the requirements of fast prediction. Therefore, the solution is applying the microscale model in the concerned local region on the background of mesoscale meteorological model. This method is called the coupling model method. Models developed on computational fluid dynamics (CFD) method can be used to simulate the local wind field on a fine grid. Several meteorological CFD models have been founded, such as WindSim of Norway and Meteodyn WT of France [3][4].

The coupling method is derived from the idea of the regional model developed by Richardson [5]. The advantage of the coupling method is that the two models that are based on the coarse grid and the fine grid respectively can be independently programmed and launched. Between the two models it only needs to build a reasonable coupling scheme to achieve the exchange of information, thus helping to improve the efficiency and accuracy of prediction. In recent years, along with the air pollution problem becoming the international issue, the method coupling the mesoscale model and microscale CFD model has widely been applied in the simulation of urban atmospheric flow and environmental pollution flow. M. Tewari [6] calculated the wind speed and direction above the Salt Lake City via coupling WRF and CFDUrban method. Wyszogrodzki [7] simulated the pollutant dispersion of Oklahoma City by coupling WRF model with a CFD-LES solver. Kwak [8] did the air quality simulation in a high-raise building area of Seoul by coupling a CFD model with mesoscale meteorological and chemistry-transport model. In the field of wind energy, Katurji [9] simulated the turbulent flow of complex terrain by coupling the WRF model and WindSim model. Gopalan [10] compared several different CFD models by coupling them with the WRF model. The results showed that the CFD model can refine

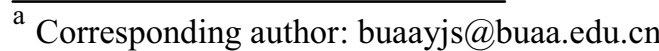


the flow structure and simulate the unsteady variation of the vortex in the wind field. Emeis [11] suggested that turbulence, thermal convection and surface-induced secondary circulations could be the three main challenges to mesoscale-microscale models.

The speed of wind is naturally much lower than the speed of sound. Assuming that the wind velocity does not influence the density of flow, the simulation will adopt the incompressible flow control equations, so that the pressure and velocity can be solved separately [12]. For the wind field prediction, it's more suitable to use the compressible flow control equations due to the severe changes in air density. Yet in order to avoid the apparent decrease of convergence rate and accuracy, i.e. 'stiffness' problems, while solving the compressible equations, the preconditioning for equations is necessary. The preconditioning method has been well developed and been widely used in solving a variety of low speed flow problems [13][14].

Taking the advantage of CFD's ability of accurately simulating the flow details and using the preconditioning method for the compressible Navier-Stokes equation of the low speed flow, this paper developed a fine mesh method for local wind field prediction. A coupling process of the CFD model and the mesoscale weather forecast model WRF is established. The coupled model carries out a refinement on the coarse mesh in WRF. A special method for information exchange on the lateral boundary of two models is adopted. The wind speed near Hong Gang-zi in Jilin, China wind power station is calculated as an application of this coupled model. The results are compared with the results generated by WRF and the measured data of wind towers.

\section{Method: Forecast model and coupling process}

\subsection{Dynamics equation}

WRF model uses the dynamics equations built in the $p-\sigma$ coordinates, the output results will be transformed into spherical coordinates of the earth. Due to the small computational area of the regional wind field, the curvature of the earth can be neglected, and thus the CFD model can be established in local Cartesian coordinate. Considering the external force, the dynamics equations of the compressible NS equations is as follows:

$$
\frac{\partial \mathbf{q}}{\partial t}+\nabla \cdot \mathbf{F}=\nabla \cdot \mathbf{F}_{v}+\mathbf{s}
$$

where $\mathbf{q}=\rho, \rho u, \rho v, \rho w, E^{T}$ is the conserved variables, $\mathbf{s}=0, f \rho v,-f \rho u,-\rho g, 0^{T} \quad$ is the source term, $f=2 \Omega \sin \varphi$ is the Coriolis force and $g$ is gravitational acceleration.

If a reference pressure $p_{0}$ is specified, the difference between the pressure of a certain point and the reference pressure, is called the perturbation pressure, denoted as $p^{\prime}=p-p_{0}$. At this time, the state equation can be written as:

$$
p=p^{\prime}+p_{0}=\rho R T
$$

Among dozens of parameterize schemes of turbulence, two Reynolds-averaged Navier-Stokes turbulence models are considered. One is B-L model. This model is an algebra model, which does not need to introduce extra control equations as well as costs small amount of calculation. It especially suits for the simulation of near wall turbulent flow [15]. The other approach to parameterize the turbulence is using the k- $\omega$ SST model. This model solves two dynamic equations, one is the equation for turbulence kinetic energy, $\mathrm{k}$, the other is for turbulence dissipation rate, $\omega$. This model is more complex than the B-L model, but it is suitable for all kinds of turbulence including near wall flow and shear flow [16].

\subsection{CFD discretization scheme based on the preconditioning method}

In the case of low speed flow, the NS equations will appear the 'stiffness' problem of slow convergence rate and poor accuracy. In order to overcome this problem, the preconditioning for the equations is necessary. For the change of wind speed within the $24 \mathrm{~h}-72 \mathrm{~h}$, it needs to adopt a dual time step algorithm for calculating the unsteady time integration. A pseudo time $\tau$ is introduced and the preconditioning only plays role on the pseudo time term.

According to the preconditioning algorithms, the prime variable is $\mathbf{Q}=[p, u, v, w, T]^{T}$ and the preconditioning matrix is [17]:

$$
\Gamma=\left(\begin{array}{ccccc}
\frac{1}{\varepsilon R T} & 0 & 0 & 0 & -\frac{\rho}{T} \\
\frac{u}{\varepsilon R T} & \rho & 0 & 0 & -\frac{\rho u}{T} \\
\frac{v}{\varepsilon R T} & 0 & \rho & 0 & -\frac{\rho v}{T} \\
\frac{w}{\varepsilon R T} & 0 & 0 & \rho & -\frac{\rho w}{T} \\
\frac{H}{\varepsilon R T}-1 & \rho u & \rho v & \rho w & \rho c_{p}-\frac{\rho H}{T}
\end{array}\right)(3)
$$

where the preconditioning parameter is $\varepsilon=M_{r}^{2}$, $M_{r}=\min 1, \max M, M_{0} \quad$, while $M$ and $M_{0}$ are the local Mach number and reference Mach number, respectively.

The control equation after preconditioning is:

$$
\Gamma \frac{\partial \mathbf{Q}}{\partial \tau}+\frac{\partial \mathbf{q}}{\partial t}+\nabla \cdot \mathbf{F}=\nabla \cdot \mathbf{F}_{v}+\mathbf{s}
$$

During the numerical solution of the above equations, LUSGS algorithm is used for the inner iteration along the pseudo time. The backward Euler implicit scheme is used for the outer iteration of physical time, where the convection term using implicit processing and the viscous term and the source terms using explicit processing. Inviscid flux is discretized by the Roe scheme. 


\subsection{The coupling of coarse mesh and fine mesh model}

The output file format of mesoscale prediction model WRF is NetCDF, which contains geographic coordinates of the coarse grid and physical quantities such as velocity, pressure and humidity. In this coupling model, the data of time, geographic coordinates, velocity, pressure and temperature are extracted as the required information for CFD computation. All the extracted data is stored in single files in time series. Along with the time advance, the program regularly read the file of each time, as the input to update the computational boundary condition. The working process of the coupling model is shown in Fig. 1:

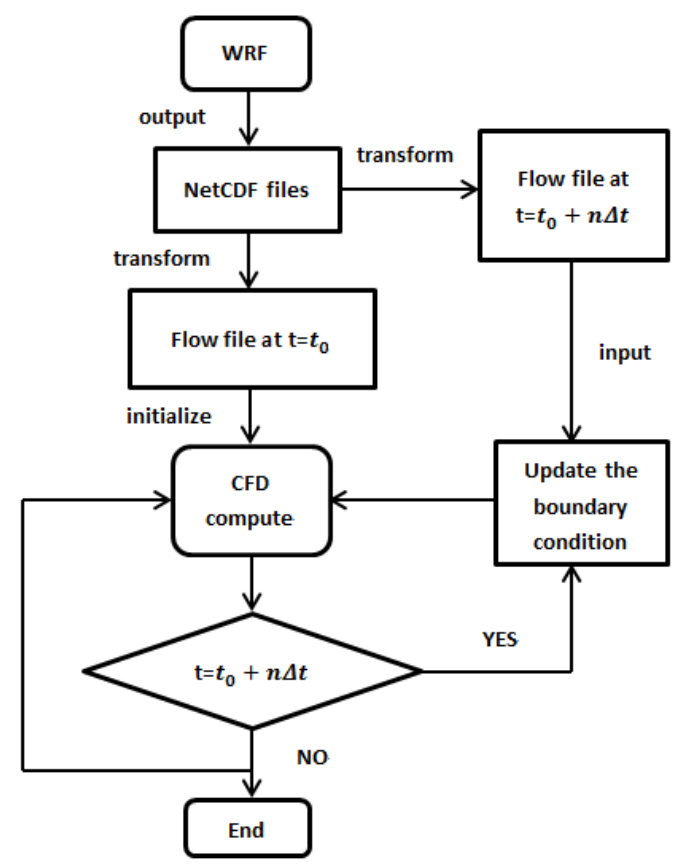

Figure 1. Working process of the coupling model.

The horizontal grid scale in the WRF model is thousands of meters, while vertical hundreds of meters. The coupling model chooses several pieces of coarse grid corresponding to the concerned local wind field to be the refining region. The refinement decreases the mesh size by one order of magnitude. Physical quantities on fine mesh point are obtained by interpolating the values on coarse mesh, of which the interpolated result in the initial time is regarded as the initial flow field for CFD simulation. The interpolated results in the follow-up moment are used as boundary conditions, to achieve the information exchange between the two models.

There are two methods to deal with boundary conditions. One is to directly use interpolated data to update the physical quantities on the boundary of the CFD calculation, which is called the fixed boundary condition. In specific, if the boundary is the flow entrance, by given the velocity and density, the temperature is obtained from interpolation of the inner field, while pressure is determined by the state equation; if the boundary is outlet, by given the outlet pressure (result from the interpolation), velocity and temperature are obtained from the interpolation of the inner field [18], while density is determined by the state equation.
To reduce the horizontal gradient of variable near the boundary, another method applied is called the transition zone method [19]. In this method, several layers of grid, i.e. the transition zone, should be added outside the computational area. In the outermost lateral of the transition zone, the fixed boundary condition is applied, while in the innermost lateral the result from CFD calculation is preserved. For the transition zone, a transition function is used for linking these two parts.

The time step of CFD is smaller than the time interval of WRF data. Therefore, during the calculation of the CFD model, at each physical time step, it needs to do the interpolation for WRF data between two adjacent moments.

\section{Numerical forecast design}

The wind field near a wind tower in Hong Gangzi of Jilin, China is selected for the wind velocity prediction. The average altitude of this area is about $130 \mathrm{~m}$. The horizontal resolution of WRF is $5 \mathrm{~km}$. 4 coarse grids around the wind tower are chosen with the total area for CFD model becoming $10 \mathrm{~km} \times 10 \mathrm{~km}$. As is seen from the computational area for the coupling model in Figure 2, the red dot is the wind tower location, while the selected grid for refinement is within the dashed-line box. Each coarse grid is divided into $20 \times 20$ fine mesh, namely a horizontal resolution of $250 \mathrm{~m}$ for CFD model. In the vertical direction, the upper boundary is taken as $1 \mathrm{~km}$ and the height of the first layer from the ground is 2 meters. The coordinates of the wind tower are 123.892 east longitude and 45.549 north latitude, in which 4 measuring points arranged at the height of $10 \mathrm{~m}, 30 \mathrm{~m}, 50 \mathrm{~m}$ and $70 \mathrm{~m}$, respectively.

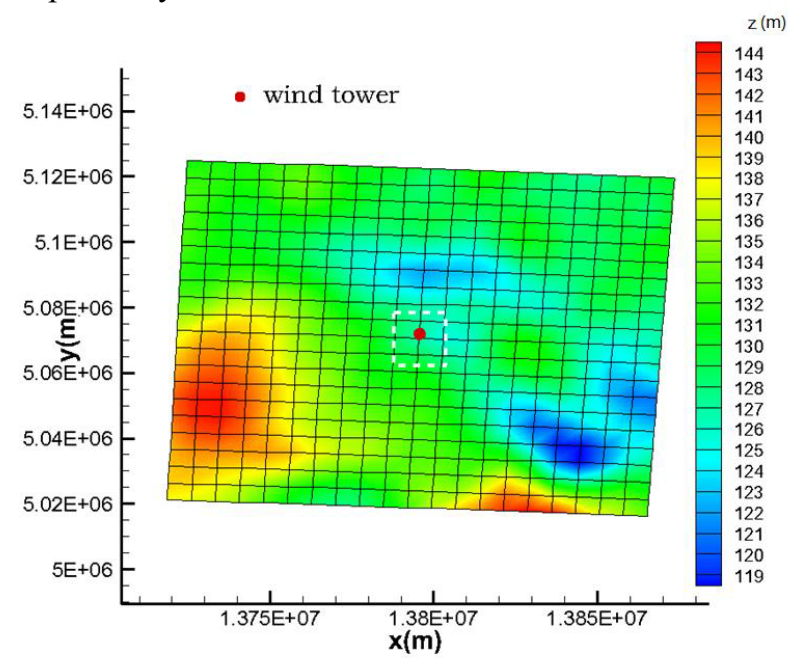

Figure 2. Computational area for the coupling model.

The whole period for a prediction is $72 \mathrm{~h}$, i.e. 3 days. WRF provides a data every 15 minutes. Hence the boundary values need to be updated every 15 minutes. A new forecast starts every $24 \mathrm{~h}$ according to the data sequence of WRF. 12:00 is chosen as the starting time for a $72 \mathrm{~h}$ forecast. The prediction process lasts from July 24th to July 29th, 2012, with a total of 6 time series (Table 1). 
For the time integration scheme, the dual-time step method is adopted. The iterative process includes the inner iteration of pseudo time and the outer iteration of real physical time marching. In order to improve the efficiency and ensure the high time accuracy, the time step of outer iteration is $20 \mathrm{~s}$, and the maximum step of inner iteration is 20 .

Table 1. Time series.

\begin{tabular}{|c|c|c|c|}
\hline $\begin{array}{c}\text { Serial } \\
\text { number }\end{array}$ & Starting time & $\begin{array}{c}\text { Serial } \\
\text { number }\end{array}$ & Starting time \\
\hline 1 & $7 / 2412: 00$ & 2 & $7 / 2512: 00$ \\
\hline 3 & $7 / 2612: 00$ & 4 & $7 / 2712: 00$ \\
\hline 5 & $7 / 2812: 00$ & 6 & $7 / 2912: 00$ \\
\hline
\end{tabular}

\section{Results and analysis}

\subsection{Results}

Fig. 3 and Fig. 4 show the instantaneous distribution of wind velocity component $\mathrm{u}$ in extracted fine mesh area at the height of about 50 meter. Comparing the WRF and the numerical results of this coupled model, it reveals that, because of the refinement of the grids and improvement of the model's accuracy, CFD model can be able to describe the flow details and characterize the gradient of the wind field variable. This feature makes it particularly suitable for processing in a region with a plurality of wind towers or wind turbines.

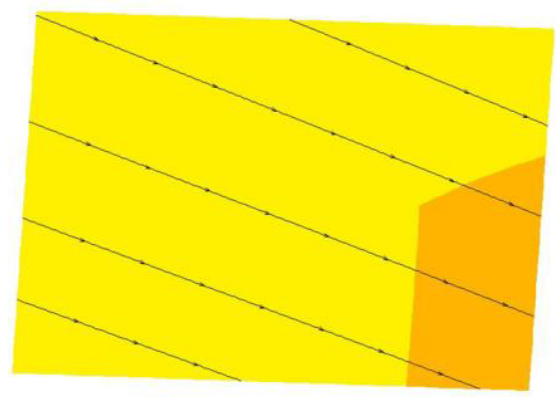

Figure 3. Counter of velocity component $u$ of WRF model.

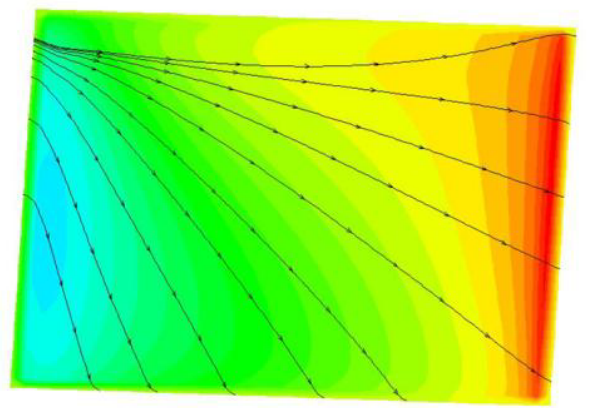

Figure 4. Counter of velocity component $u$ of CFD result.

The result of a set of time series (July 26th 12:00) at the height of $70 \mathrm{~m}$ is presented in Figure 5 and Figure 6. It can be roughly seen from the figures that, through enhancing the accuracy of the WRF model, the result of the coupling model is more close to the measured curve of wind velocity. On the other hand, the wind direction results provided by WRF and CFD model have almost the same trend.

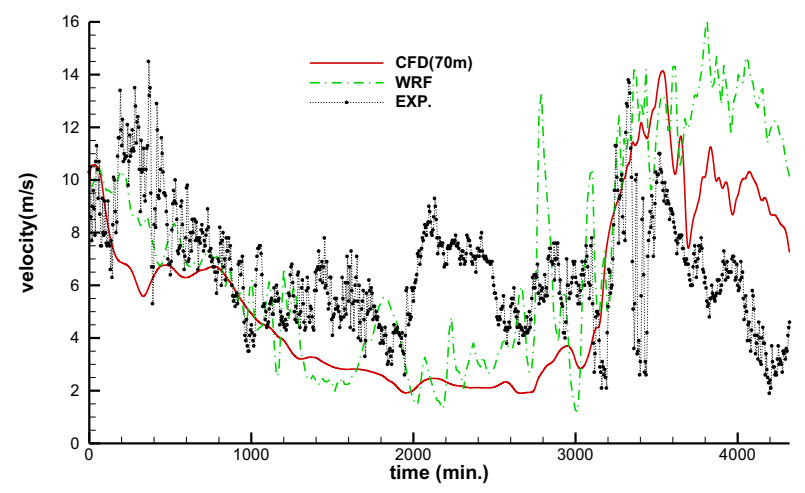

Figure 5. Wind velocity curve of 7/26 12:00 at $70 \mathrm{~m}$.

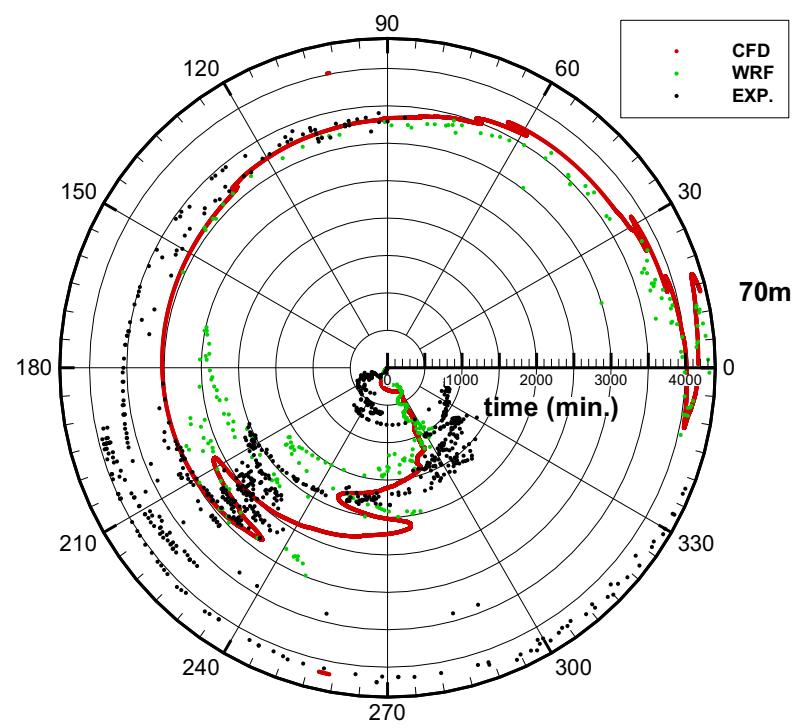

Figure 6. Wind direction curve of 7/26 12:00 at $70 \mathrm{~m}$.

Due to the results of WRF are used as the initial field and the boundary condition for CFD calculations. The results of CFD and WRF have strong similarity. It can be named as 'the following property' of the fine mesh model for the coarse mesh model. Because the WRF model is the only input of the CFD model, the wind velocity of two models increases or decreases similarly with the inflection point appearing in different positions. In fact, the CFD model corrects the position of the inflection point, so that the shape of the wind speed curve is closer to the measured data.

CFD simulation has a certain time lag effect relative to WRF data, namely 'the lag property' of fine mesh model to the coarse mesh model. That is because in each time step, the data of WRF is just input as the boundary condition for CFD calculation, which means the changes of the flow field will take time to spread from the boundary to interior of the wind field.

The CFD simulation has a certain 'smoothing' effect on the wind velocity curve, making the temporal variation of wind velocity not so intense. Results of WRF vibrate acutely at some point, seriously deviating from the measured data. CFD model can avoid wind velocity to appear large fluctuation, which improves the calculation results of WRF. By modifying the calculation 
time step, the 'smoothing' effect can be increased or decreased, to achieve the resolution adjustment according to the actual situation and the requirements.

\subsection{Parallel programming and time consumption}

Accuracy of CFD simulation requires a big amount of computation time. In order to increase the efficiency of the coupling model and meet the requirement of fast forecast, parallel programming is necessary. The OpenMP multi-thread parallel programming is adopted. By optimizing the loop calculation in the CFD simulation, this method is easy to program and can provide a considerable improvement in efficiency.

Table 2. Computation time of the coupling model.

\begin{tabular}{|c|c|}
\hline & Time(s) \\
\hline Serial & 19617 \\
\hline Parallel & 15163 \\
\hline
\end{tabular}

The parallel model is running with 6 threads. Tab. 2 reveals the time consumption before and after the parallel is adopted. The parallel gives the program an acceleration of more than $20 \%$.

\subsection{Error analysis}

In order to evaluate the optimization effect of CFD model, the average error and the correlation coefficient between the WRF and CFD results are compared with the measured data of each time series. The average error indicates the mean value of the error between computational results and measured data, while the correlation coefficient indicates the degree of the linear correlation between computational results and measured data. Computing the 6 sets of time series shown in Table 1 with B-L model, the error analysis at the height of $10 \mathrm{~m}$ and $50 \mathrm{~m}$ is carried out and the results are shown in Fig. 7 and Fig. 8, respectively.

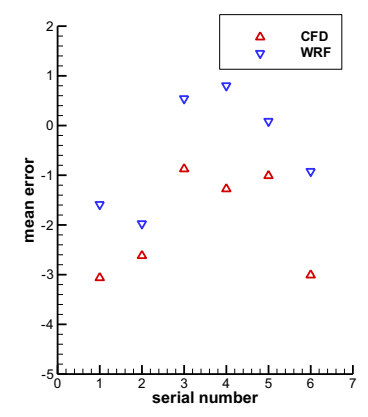

(a)

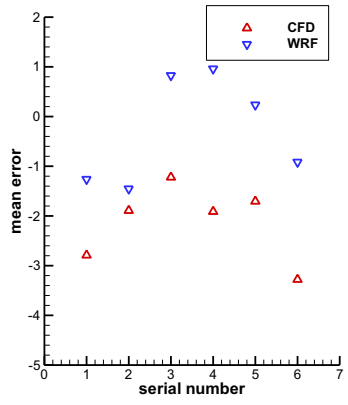

(b)
Figure 7. Mean error (a) $10 \mathrm{~m}$ (b) $50 \mathrm{~m}$.

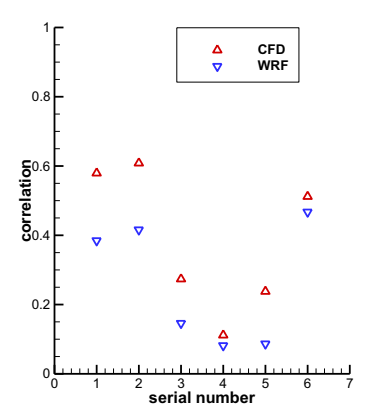

(a)

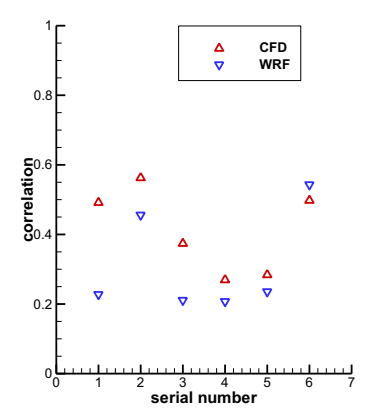

(b)
Figure 8. Correlation coefficient (a) $10 \mathrm{~m}$ (b) $50 \mathrm{~m}$.

From Fig. 7 it can be seen that, the coupled CFD model has a larger mean value than the WRF model, whereas the values are minus. It shows that in general, the wind velocity calculated by CFD is smaller than that by WRF. The near wall boundary layer effect decreases the wind speed magnitude. Therefore, the extremely high wind speed deriving from WRF can be avoided. Figure 8 shows that the coupled CFD model has lager correlation coefficient than the WRF model, which means the curve computed by the CFD model is closer to the measured data. Overall, the result of coupling model is more sophisticated, closer to the measured wind velocity and has optimized the forecast results of WRF.

\subsection{Comparison of turbulence models}

Two models are chosen for the parameterization of turbulence, one the algebraic B-L model, the other is twoequation $\mathrm{k}-\omega$ SST model. Theoretically, the algebraic operation of B-L model is efficient and fast, and it is suitable for near wall flow. However, it is not suitable for the wake flow, the shear flow, etc. SST model is a relatively well-developed two-equation model, which more accurately calculates the adverse pressure gradient and separated flow. Yet solving two extra equations, computational efficiency of this model is lower than the algebraic model. The model has to take into account the efficiency and accuracy of wind prediction. Thus the evaluation of these two models is very important. Table 3 is the comparison of the two models in the aspect of calculation time.

Table 3. Computation time of two turbulent models.

\begin{tabular}{|c|c|}
\hline & Time(s) \\
\hline B-L & 15163 \\
\hline k- $\omega$ SST & 16386 \\
\hline
\end{tabular}

The results show that for short-term forecasting, both models can achieve completing 72 hours' forecasts within 5 hours. Since the turbulence equations are not a major part of the calculation, and the parallel programming methods also have a significant impact on the calculation speed, B-L mode computing speed is not much faster than the k- $\omega$ SST model. From this point of 
view, by introducing of parallel computing, slow shortcomings of two-equation k- $\omega$ SST model calculations can be made up.

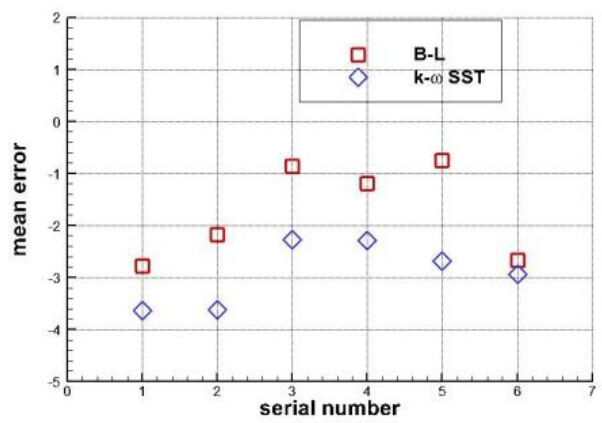

Figure 9. Mean error of different turbulent models.

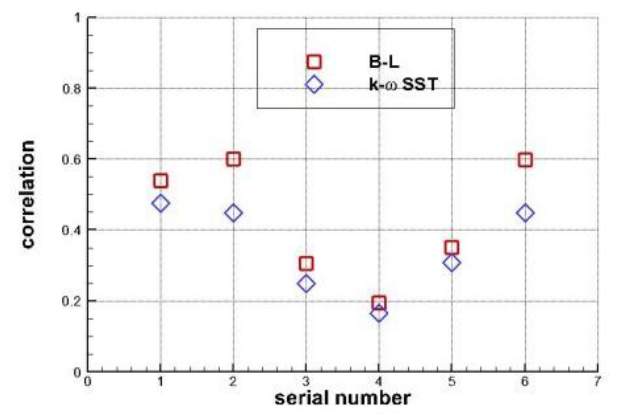

Figure 10. Correlation coefficient of different turbulent models.

Fig. 9 and Fig. 10 further compare the average error and the correlation coefficient of B-L model and k- $\omega$ SST model. It can be seen from the figures that, compared to the k- $\omega$ SST model, the B-L model is more accurate. B-L model is an algebraic model, so in each calculation step it directly gives the exact value of the turbulent viscosity coefficient. While in $\mathrm{k}-\omega$ SST model the accurate obtaining of turbulent viscosity coefficient requires a lot of iteration, and the excessive number of iteration will reduce the efficiency. Thus, considering the computational efficiency and accuracy, for this coupling mode, B-L model is a better choice. However, the terrain of this forecast is relatively flat. Whether k- $\omega$ SST model will perform better in the undulating terrain with mountains still needs to be verified.

\section{Conclusion}

The author has established a CFD model suitable for microscale wind field simulation. This model uses the preconditioning technique to solve the compressible NS equations, parameterizes the turbulent viscosity coefficient with the BL model and k- $\omega$ SST model. On this basis, by coupling the CFD model and WRF model, a nested model which can realize the fine forecast for local wind field is developed. The coupled model is successfully applied in the wind field prediction near a wind tower in Hong Gang-zi, Jilin, China. The prediction results are compared with that of the WRF model and the measured data.

The CFD model can be quickly coupled with the WRF model, and can carry on the delicate simulation of the local wind field, to describe the wind field details.
According to the results of a series of time sequence, as the fine grid model, the CFD model can effectively augment the prediction precision of the WRF model. The wind velocity curve of the coupled model is overall close to that of WRF, presenting 'the follow property', 'the lag property' and 'smoothing' effect. These characteristics of CFD model modify the inflection point and smoothness of WRF model curve, thus make it closer to the measured data. In addition, as can be seen from the error analysis, CFD model can significantly reduce the calculation error of the WRF model.

The established CFD and WRF coupling model can undertake delicate simulation for the wind field above local complex terrains and can be used for short-term wind velocity forecast. In order to improve the accuracy of CFD model and more accurately describe the velocity fluctuations, the next research direction will be to reduce the coupling degree, optimize the iterative scheme of time integration, to optimize the turbulence parameterization schemes, and to consider the land surface process.

\section{References}

1. M. Jannatia, S. H. Hosseiniana, B. Vahidi, Renew. Sust. Energ. Rev. 29 158-127( 2014)

2. A. Gsella, A. de Meij, A. Kerschbaumer et al, Atmos. Environ. 89 797-806( 2014)

3. S. Milashuk, W. Crane, Environ. Mod. Soft. 26 429-433(2011)

4. J. Manning, P. Hancock, R. Whiting, Wind Eng. 5 477-500(2010)

5. L. Richardson, Weather Prediction by Numerical Process(London: Cambridge University Press, 1922)

6. M. Tewari, H. Kusaka, F. Chen et al, Atmos. Res. 96 656-664(2011)

7. A. Wyszogrodzki, S. Miao, F. Chen. Atmos. Res. 118 324-345(2012)

8. K. Kwak, J. Baik, Y. Ryu et al, Atmos. Environ. 100 167-177(2015)

9. M. Katurji, (Ph. D. thesis, University of Canterbury, (2011)

10. H. Gopalan, C. Gundling, K. Brown, J. Wind Eng. Ind. Aerod. 132 13-26 (2014)

11. S. Emeis, J. Wind Eng. and Ind. Aerod. 144 24-30(2015)

12. C. Bruneau, M. Saad, Comput. Fluids, 35 326348(2006)

13. E. Turkel, Appl. Numer. Math. 12 257-284(1993)

14. Y. Colin, H. Deniau, J. Boussuge, Comput. Fluids, 47 1-15(2011)

15. B. Baldwin, H. Lomax, Aerospace Sciences Meeting, $16^{\text {th }}(1978)$

16. F. Menter, I. Rumser, 25th AIAA Fluid Dynamics Conference, Colorado(1994)

17. C. Merkle, P. Buelow, J. Sullivan et al, AIAA J. 36 515-521(1998)

18. E. Turkel, Ann. Rev. Fl. Mech. 31 385-416(1999)

19. A. McDoland, PINSA, 65 91-105(1999) 